\title{
Entropy and the city
}

\author{
N. Marchettini, F. M. Pulselli \& E. Tiezzi \\ Department of Chemical and Biosystems Sciences, \\ University of Siena, Italy
}

\begin{abstract}
The sustainability assessment of a complex human system, such as a city, must start from the knowledge of physical laws and constraints which the development of the system is based on. It is well known that the Second Law of thermodynamics states that an isolated system tends towards maximum entropy, that implies maximum disorder. In general, life seems to contradict the Second Law since we observe that the trend of living organisms is towards the creation of order where previously there was disorder: it is the trend to organize and self-organize. Ilya Prigogine called these systems "dissipative structures" that are thermodynamic self-adaptive systems open to both energy and matter. Human systems (economic, social, urban, etc.) use relations and exchanges with their environment in order to survive and might be identified as dissipative structures far from thermodynamic equilibrium. In particular, the evolution and maintenance of a city is possible due to inputs of natural resources from the terrestrial ecosystems and outputs of heat and wastes into terrestrial ecosystems. Edgar Morin said that the autonomy of a system is funded on the dependence on the environment so that autonomy and dependence are complementary concepts, although they seem in contrast, and there cannot be autonomy without dependence. Different kinds of inputs, especially from the environment, are stored in a city and become part of its "living" structure. Morin's concepts of autonomy and dependence for human systems suggest that those systems must evolve between two extremes: autarchy and globalisation. This paper seeks to show how, in entropic terms, these two configurations are inconsistent with the concept of sustainability.
\end{abstract}

Keywords: sustainable development, second law, entropy, autarchy, globalization. 


\section{Introduction: a thermodynamic framework}

The term entropy, indicated with $\mathrm{S}$, was coined by Clausius from $\tau \rho o \pi \eta$ (transformation) and $\varepsilon v \tau \rho o \pi \eta$ (evolution, mutation or even confusion). For an isolated system $\Delta \mathrm{S} \geq 0$, equality holds only for ideal reversible processes (e.g. a Carnot cycle). In an ideal reversible transformation between two states of a system, entropy variation is given by the sum of the ratios between heat quantities, exchanged with the environment in a succession of short whiles during the transformation process, and the absolute temperatures (considering infinitesimal whiles, thus infinitesimal heat quantities, this sum is given by a sum of integrals).

Thus, in classical thermodynamics, entropy is a state function whose variation, in the transition of a system from a state to another, can be calculated.

In real irreversible transformations of an isolated system, according to the Second Law of Thermodynamics, the entropy variation is always positive, thus entropy tends to a maximum, that is the end of spontaneous evolution of the system. Any real process can only proceed in a direction which results in an entropy increase. Heat always flows spontaneously from a hotter reservoir to a colder one, until there is no longer a temperature difference or gradient; gas always flows from high pressure to low pressure until there is no longer pressure difference or gradient. This principle has been applied to the whole universe suggesting the hypothesis of a trend towards a thermal death; it represents the extent to which nature becomes more disordered or random. Entropy is also an indicator or even an evidence of the existence of time (the arrow of time) because it gives a direction to the succession of states of a system.

It is necessary to distinguish between isolated systems (which cannot exchange energy or matter with the outside world), closed systems (which can exchange energy but not matter, e.g. our planet) and open systems (which can exchange both energy and matter). Cities and biological organisms are examples of open systems. For such systems we must sum the negative entropy produced inside the system with the positive entropy created in the environment. We then see that if "sometimes disorder degenerates into order" this is only a facade, the appearance of order at the price of even greater disorder in the surrounding environment. Living systems therefore need a continuous flux of negative entropy from the universe, to which they return an even larger amount of positive entropy. Ilya Prigogine called these open systems "dissipative structures".

Now we have a suitable formulation and a powerful tool to understand how the entropy variation in open systems is the sum of two terms: external entropy fluxes and internal entropy production.

From a thermodynamic viewpoint, any ecosystem is an open system far from thermodynamic equilibrium, in which entropy production is balanced by the outflow of entropy to the environment. The climax of the ecosystem corresponds to a dynamic equilibrium (steady-state), when the entropy production inside a system is balanced by the entropy flow from the system to its environment. 
In the theory of open systems and dissipative structures by Prigogine, as we have seen above, the total variation of entropy is presented in the form of two items:

$$
\mathrm{dS}=\mathrm{d}_{\mathrm{i}} \mathrm{S}+\mathrm{d}_{\mathrm{e}} \mathrm{S} \quad \mathrm{d}_{\mathrm{i}} \mathrm{S}>0
$$

where $\mathrm{d}_{\mathrm{i}} \mathrm{S}$ is the entropy production (heat, disorder) caused by irreversible processes within the system and $\mathrm{d}_{\mathrm{e}} \mathrm{S}$ corresponds to the entropy of exchange processes between the system and its environment; it is the transfer of entropy across the boundaries of the system.

In this formulation, the distinction between irreversible and reversible processes is essential. Only irreversible processes produce entropy. The Second Principle therefore states that irreversible processes lead to a sort of unidirectional time. For isolated systems $\mathrm{d}_{\mathrm{e}} \mathrm{S}=0$ and the previous equations becomes the classical Second Law. Open systems could conceivably evolve to steady states with:

$$
\mathrm{d}_{\mathrm{e}} \mathrm{S}=-\mathrm{d}_{\mathrm{i}} \mathrm{S} \quad \mathrm{dS}=0
$$

This is a non-equilibrium steady state that should not be confused with thermodynamic equilibrium, and in which order may be created from disorder. Order created in this way no longer violates the laws of thermodynamics; equilibrium is no longer the only attractor of the system, but the world becomes more complex and thermodynamics can embrace new worlds characterized by highly organized as well as chaotic structures.

In this way thermodynamics' second principle addresses the pathways we should avoid in order to keep life on Earth. It shows the universal, inescapable tendency towards disorder (or the general trend toward an entropy maximum), which is also a loss of information and of usable energy availability.

Living systems try to avoid the condition of thermodynamic equilibrium, keeping themselves as far as possible from that state, self-organizing due to material and energetic fluxes, received from outside and from systems with different conditions of temperature and energy. However, even biological systems, ecosystems and especially human systems can suffer this tendency to the Clausius" "thermal death" through the destruction of diversities [1]. There are two ways to achieve such a condition:

a) when, through energy exchanges as heat fluxes, there are no more differences in temperature and nothing more can be done, because any exchange of usable energy is allowed;

b) when a system, becoming isolated, consumes its resources, reaching a great increase in its internal entropy and, at the end, to self-destruction.

Economics and our society cannot be unaware of thermodynamics' second principle. As a consequence globalization, the destruction of both biological and cultural diversities, homogenization and the unique thought take inescapably to the thermal death. In the same way a country, a nation, a system that makes a political dogma of its isolation, of its refusing of cultural contamination (better: 
of cross-fertilizations), of its castling on fundamentalist positions of selfconservation, will go to the same end. An excessive defence of one's diversity and a complete loss of diversity are two faces of the same thermodynamic violation.

\section{Urban systems, complexity and entropy}

An urban system is a both complex and complicated system, and therefore not completely determinable. Dynamic phenomena and surprising new events spring from the variable relationships between the parts and from collective behaviours that escape individual control. The evolution of urban systems is governed by irreversible and stochastic processes combining choice and chance. Clear signs may be recognised, for example the way in which certain technologies have recently changed the way people move and exchange information, and how they use public and private urban space. Mobility, telecommunications, energy and material flows that feed a city raise practical evolving questions in the field of urban studies.

Emergent landscapes have changed the perception of cities, drawing attention to general behaviours and ordering principles in a holistic framework. The idea that a city can be conceived as a complex self-adapting system, or even a living ecosystem, is a key point for urban research and opens promising perspectives to direct strategic choices in future development. Certain concepts, from complex system theory to evolutionary physics, from thermodynamics to ecology, confirm this hypothesis and are in line with Eugene Odum's definition of an ecosystem as a unit of biological organization consisting of all the organisms in a given area interacting with the physical environment [2].

The interactions between society and the built environment, living and nonliving systems, change and take various forms that can be observed on different space-time scales. Organizational and developmental properties of cities and ecosystems must be considered as wholes and their structural and dynamic properties studied in order to describe and explain the formation of macro-level patterns in systems composed of many interacting micro-level components [3]. An ecosystem approach is required if we assume that some of the properties and behaviours of certain urban systems depend on interactions between their parts and with the surrounding environment. A proper understanding of these properties and behaviours will require bringing these system-environment relations explicitly within the field of investigation [4].

This ecosystemic approach to the study of anthropic (urban) systems can be well represented by the use of the energy system language proposed by Howard T. Odum [5]. An energy diagram gives a synthetic description of resource flows and transformation processes that take place in the territory; it works as a scheme in which relationships between the system and the outside and between its own parts have been decoded in the form of flows of energy and materials. Specifically, it provides a comprehensive glance on urban and regional dynamics based on the ability to group different aspects of a territory and different sectors into a unique vision. 
A city is part of a much vaster territory and many processes have a wide range of action or, even, a global dimension. Without continuous flows of input energy that build order, these systems would degrade. The inventory of the inputs that supply a city comes from an observation of multiple dynamics and processes. System diagrams provide a holistic picture of the ecosystems, or complex systems, specifying the main forcing functions, internal components, process interactions, etc. [6]. Diagrams are characterised by geographic boundaries and the fundamental elements within them: external sources, natural deposits or storages, primary producers, human activities and settlements (industries and cities, respectively) and institutions that communicate by flows of energy, matter, information, money, people, wastes and heat (fig. 1).

Evolutionary physics and non-equilibrium thermodynamics are the main disciplines for studying dynamics of complex systems and self-organization in urban social systems. Prigogine [7] underlines that cities and living organisms embody different types of functional order. "To obtain a thermodynamic theory for this type of structure we have to show that non equilibrium may be a source of order. Irreversible processes may lead to a new type of dynamic states of matter which I have called "dissipative structures". [...] These structures are today of special interest in chemistry and biology. They manifest a coherent supermolecular character which leads to new quite spectacular manifestations."

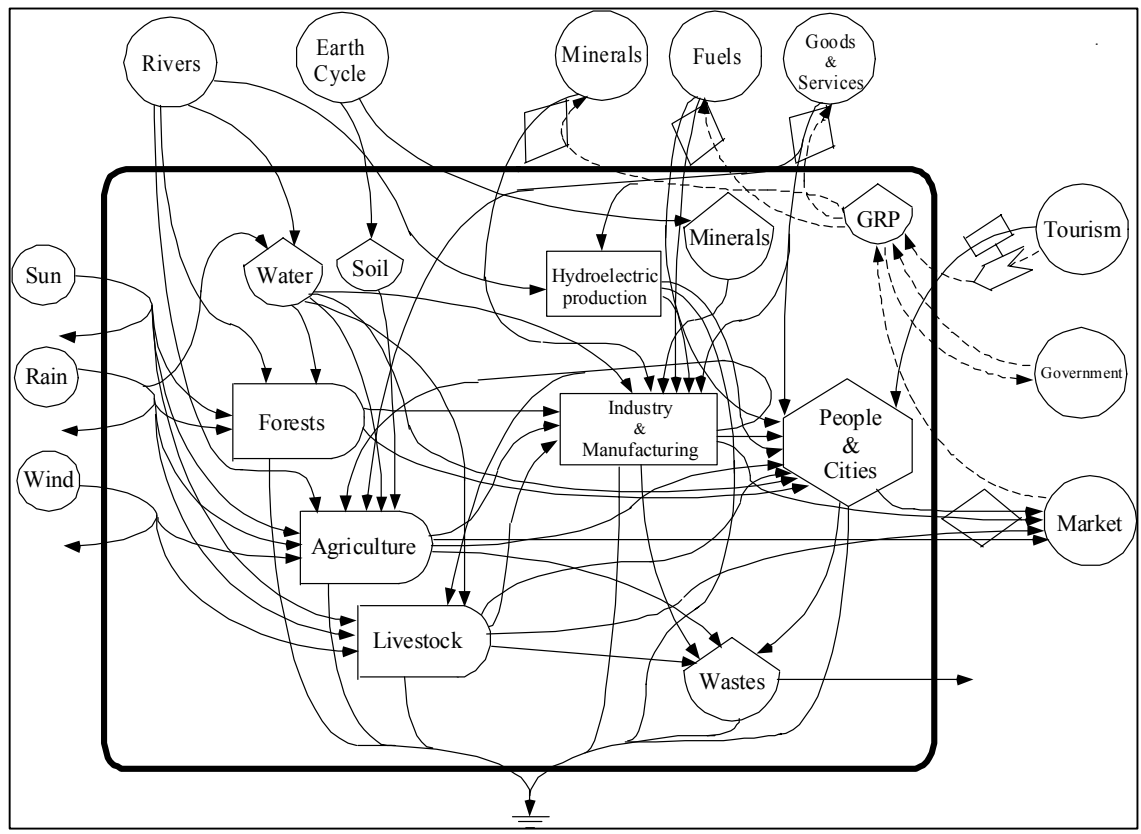

Figure 1: The energy diagram is a schematic description of the flows of resources and transformation processes within and through the given boundaries of the system. It is a synthetic idea of the relationships between elements and processes in form of fluxes of energy and matter. 
How can a city be conceived as a dissipative structure?

Absorption of external input (negentropy) and emission of internal output (entropy, heat) is a principle that works for social systems, economics, human settlements and all their dynamics. Cities absorb flows of high quality energy from the external environment and emit heat, wastes and pollutants; their internal entropy decreases by self-organization in the form of structures, information, social patterns and economy. Resource flows feed these dissipative cities, as if they were ecosystems composed of organisms and are metabolized and continuously used to sustain its ordered structure during the time.

Entropy change depends on two processes: interactions among constituents of the system $d_{i} S$ and exchanges with the external environment $d_{e} S$ (see above). How can we describe these processes in a complex urban system?

The configuration of a complex system, or organization of parts into a whole, is therefore a steady state and depends on interactions among constituents of the system (responsible for the entropy change $\mathrm{d}_{\mathrm{i}} \mathrm{S}$ ) and perturbations from the external environment (responsible for the entropy change $d_{e} S$ ). The configuration is always dynamic: it is not static in time but changes whenever external conditions change.

\section{Biophysical foundations}

Cities are physical systems in contact with various sources and sinks; matter and energy flow through them from the sources to the sinks.

Let us consider the flow diagram [8]:

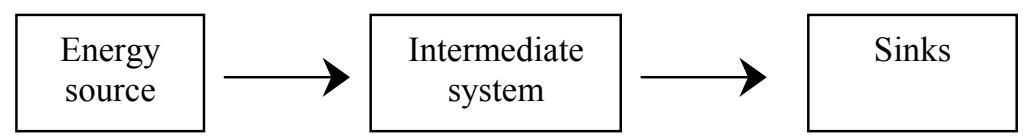

and divide the system into two parts:

1. $\quad \operatorname{source}(s)+\operatorname{sink}(s)$

2. intermediate system(i).

According to the Second Law of Thermodynamics:

$$
\mathrm{dS}_{\mathrm{ss}}+\mathrm{dS}_{\mathrm{i}} \geq 0
$$

where $S_{s s}$ is the entropy of source + sink and $S_{i}$ is the entropy of the intermediate system. The flow of energy from the source to the sink always involves an increase in entropy:

$$
\mathrm{dS}_{\mathrm{ss}}>0
$$

whereas the only restriction of the second law on $\mathrm{dS}_{\mathrm{i}}$ is that:

$$
\mathrm{dS}_{\mathrm{i}} \leq \mathrm{dS}_{\mathrm{ss}}
$$


so that the entropy of the intermediate system (in our case a city) may decrease if there is an energy flow. A flow of energy provides the intermediate system with quantities of energy for the creation of far-from-equilibrium states, that is, states far from thermal death.

Two related elements must be put on evidence:

I) as we have already quoted, a source of low entropy is as necessary as the sink into which high entropy must be discharged;

II) it is the continue flow through the system and not the mere availability of resources that enables it to survive, develop and increase in complexity.

The transition from a low entropy input to high entropy output describes the evolution of a far from equilibrium system as a dissipative structure and the irreversibility of such a pattern according to the Prigogine's concept of arrow of time [9].

The evolutionary process is such that systems become more and more complex and organized. Biological diversity is the key element that derives from long term interactions at a genealogical and ecological level, the former regarding the dissipation of entropy by irreversible biological processes and the latter regarding entropy gradients in the environment.

Morowitz's scheme suggests that the inhibition of the flow of low entropy from sources to sinks could jeopardize the capacity of a system to survive in the long run. Furthermore, the intermediate system is an anthropic system and the amount of resources involved in its processes is correlated to its dimensions. The larger is the system and the more "urgent" are both the supporting inputs (low entropy) and the capacity of discharging wastes (high entropy). Hence, the fundamental biophysical bases for an urban system like a city to survive indefinitely rely on its consistency with physical limits imposed by thermodynamics and the preservation of ecological surroundings and, consequently, of ecosystem services.

In this context, Morin [10] defines the concept of autonomy as funded on the dependence on the environment. Autonomy and dependence are complementary concepts, although they seem in contrast, because a system needs resources to self-organize and maintain its individuality and originality. Hence selforganization and eco-organization have to be interconnected and this is a conceptual problem of complexity. There cannot be autonomy without dependence.

Once the dissipative nature of anthropic processes and their dependence on natural sources and sinks have been acknowledged, the management of economic as well as urban systems is a problem of decision making, that should be oriented according to the above statements.

\section{Extreme evolutionary patterns}

Like a living system, an anthropic system must develop by mediating between two extreme situations, namely autarchy and globalization, that are both unsustainable from a thermodynamic point of view. Theoretically speaking, perfect autarchy and globalization are arbitrary concepts and are to economics as 
the concept of absolute vacuum is to physics and that of isolated system is to thermodynamics. They are scholastic representations of hypothetical economic realities that do not exist, but they are useful as hypotheses for human life.

The idea of an autarchical human system is related to a sort of selfconsistency. In general, the concept is referred to political systems as selfgovernment and to economic systems as self-sufficiency. Autarchical is a closed socio-economic system that feeds upon its internal resources without trading with the outside. It is neither economically nor culturally influenced by external systems. The lack of relations with other systems and cross fertilization experiences makes autarchy a rare and unnatural condition, often driven by a dictatorship. As a system, it is composed by subsystems that are often forced to avoid any connection with external elements. An autarchical system is assimilable to an isolated system, with no exchanges of energy, matter, information, culture etc. with the outside. Like for biological systems, the ordered state of such a system would decay, if left to itself, towards the most disorderly state possible. In order to avoid the condition of maximum entropy, in fact, a system may continuously export the produced entropy to other systems so that, even in a steady state, i.e. in case of constant entropy, the entropy of the total (system plus surroundings) increases, according to the Second Law. In condition of autarchy both the inflow of negentropy and the export of high entropy are inhibited, contravening the observations I) and II) seen above and driving the system to the exhaustion of its vital processes.

Globalization process is, in general, the increasing integration of international markets with the major priority of promoting economic growth. The concept of "integration", typical of globalization, goes beyond the simple "interdependency" of national economies which the process of internationalization is based on. It is the effective erasure of national boundaries for economic purposes, with social and cultural implications. Distribution inequality, together with different working conditions, poverty and lack of democracy somewhere, makes the markets of different countries not comparable with each other and a global economy difficult to realize. Each one considers the "other" markets within an unilateral vision of globalization: new consumers to stimulate and fresh energy and matter to exploit. Globalization is a growing, and apparently endless, process, whose keywords are efficiency, competency, ability, flexibility and competitiveness. It induces the global economy to maximize some crucial magnitudes, first of all consumption and production. Thermodynamics does not justify the project of an infinite growth in a finite planet and the level of exploitation of natural resources, including the capacity of ecosystems to absorb anthropic emissions, is already beyond the carrying capacity of Earth. The growing demand of energy and matter to support the global system and the eventual presence of an adiabatic membrane around the planet (increasing greenhouse effect) imply that globalization contravenes the observation I) and II) derived from the Morowitz's entropic scheme.

In Morin's terms of autonomy and dependence, the growing integration of international markets, together with cultural homologation and loss of diversities, is generating a huge organism (the global system) more and more dependent on 
the availability of ecosystem's goods and services that, in turn, are becoming more and more scarce. When the capacity of Earth of providing goods and services is completely exhausted, then the maximum level of globalization will be paradoxically the extreme form of economic autarchy.

It is our opinion that autarchy and globalization are two extreme configuration of an economic, but also socio-political, system whose evidences are not consistent with physical laws, in particular the entropy law.

\section{Conclusions}

Mankind is similar to other biological species. Socio-economic and urban systems are living subsystems within the greater system-biosphere: they can evolve like biological systems but their evolutionary processes are much more rapid than the capacity of the environment to respond to such changes. This could lead to unpredictable consequences for all living species and for the environment. The paper shows the necessity to manage the thermodynamic limits to growth of an anthropic system in order to avoid the risk of incurring in an "entropic euthanasia". Two extreme configurations were described that imply the risk of a thermal death: (a) autarchy: similar to the condition of a thermodynamic isolated system destined to the maximum entropy as the Second Law of thermodynamic states; (b) globalization: the unlimited increasing of quantitative flows of energy and matter to feed a larger and larger structure, that is to say that maximum level of globalization corresponds to a maximum homologation, no more differences between the subsystems of global system, no more potentialities to develop, hence maximum disorder and maximum entropy. The development of an urban system, as a dissipative structure strongly dependent on continuous flows of energy and matter from the environment, has to follow a path between those two extreme vicious and convergent configurations.

\section{References}

[1] Tiezzi, E. The essence of time. Wit Press, Southampton, 2002.

[2] Odum, E. The strategy of ecosystem development, Science, 164, 262-270, 1969.

[3] Ulanowicz, R.E. Growth and Development: Ecosystems Phenomenology, Springer-Verlag, New York, 1986.

[4] De Laplante, K. and Odenbaugh, J. What isn't wrong with ecosystem ecology, in: R.A. Skipper, C. Allen, R. Ankeny, C. F. Craver, L. Darden, G. M. Mikkelson, and R. C. Richardson, eds., Philosophy of the Life Sciences, MIT press, Cambridge, USA, in press, 2006.

[5] Odum, H.T. Environmental Accounting, Emergy and Environmental Decision Making. Wiley \& Sons, New York, 1996.

[6] Tilley, D.R., Swank, W.T. Emergy-based environmental system assessment of a multi-purpose temperate mixed-forest watershed of the 
272 The Sustainable City IV: Urban Regeneration and Sustainability

southern Appalachian Mountains USA. Journal of Environmental Management, 69, pp. 213-227, 2003.

[7] Prigogine, I. Time, Structures and Fluctuations, Nobel Lecture, 1977.

[8] Morowitz, H. Energy flow in biology. Ox Bow Press, Woodbridge, Connecticut, USA, 1979.

[9] Prigogine, I. The arrow of time. In: C. Rossi, and E. Tiezzi (Editors) Ecological Physical Chemistry. Elsevier, Amsterdam, NL, pp. 1-24, 1991.

[10] Morin, E. Le vie della complessità. In: G. Bocchi and M. Ceruti (Editors), La sfida della complessità. Feltrinelli, Milano, Italy, pp. 49-60, 1995. 